INRA Prod. Anim.,

2013, 26 (1), 25-34

\title{
Les rôles des caractéristiques sensorielles des aliments dans le comportement alimentaire des ruminants domestiques
}

\author{
A. FAVREAU-PEIGNE' $E^{1,2,3,4}$, R. BAUMONT ${ }^{1,2}$, C. GINANE $E^{1,2}$, \\ 1 INRA, UMR1213 Herbivores, F-63122 Saint-Genès-Champanelle, France \\ ${ }^{2}$ Clermont Université, VetAgro Sup, UMR Herbivores, BP 10448, F-63000 Clermont-Ferrand, France \\ 3 INRA, UMR791 Modélisation Systémique Appliquée aux Ruminants, F-75005 Paris, France \\ ${ }^{4}$ AgroParisTech, UMR Modélisation Systémique Appliquée aux Ruminants, F-75005 Paris, France
}

Courriel : angelique.favreau@agroparistech.fr

Simples agents permettant de discriminer les aliments ou bien agissant directement sur les préférences et l'ingestion des animaux, le rôle des caractéristiques sensorielles sur le comportement alimentaire des ruminants est encore largement débattu dans la littérature. Des études récentes permettent de mieux connaître leurs multiples rôles et leurs intérêts pour les ruminants selon la complexité de l'environnement alimentaire.'

Au pâturage, les ruminants sont confrontés à des contextes d'alimentation très variés, depuis les prairies semées d'une seule espèce jusqu'aux prairies permanentes hautement diversifiées. Ils y rencontrent une importante variabilité d'items alimentaires qui diffèrent en qualité et en disponibilité selon les espèces végétales présentes, leur stade de développement et leur aspect général (hauteur, humidité...). En bâtiment, les ruminants sont également confrontés à une diversité alimentaire, même si c'est dans une moindre mesure, puisqu'ils se voient généralement offrir un ou deux fourrages ainsi que des aliments concentrés. Même si les différents composants de la ration sont souvent mélangés dans une ration complète, la diversité alimentaire peut continuer à jouer un rôle dans le comportement alimentaire des ruminants du fait de leur capacité à trier et sélectionner les aliments (Dumont 1997).

Dès lors que les ruminants sont face à une offre alimentaire diversifiée, il est essentiel qu'ils soient capables de sélectionner une alimentation adaptée à leurs besoins, en qualité et en quantité. Ils disposent alors de deux types d'informations pour évaluer les aliments : i) les informations pré-ingestives liées aux caractéristiques sensorielles des aliments perçues par l'animal avant d'avaler l'aliment et ii) les informations post-ingestives liées aux conséquences digestives et métaboliques ressenties par l'animal après avoir avalé l'aliment. Ces informations sont ensuite intégrées par l'animal pour évaluer ce qui est souvent appelé la palatabilité ou l’appétibilité de l'aliment.

La palatabilité est un concept clé pour comprendre comment les informations pré- et post-ingestives interagissent, mais elle connaît de nombreuses définitions rendant son utilisation parfois difficile (Baumont 1996, Forbes 2010). Greenhalgh et Reid (1971) ont suggéré que la palatabilité reflète les caractéristiques des aliments qui provoquent une réponse des sens de l'animal. D'après Rogers (1990), la palatabilité est une construction hypothétique nécessaire pour expliquer la valeur hédonique des caractéristiques sensorielles des aliments ; la palatabilité est d'abord influencée par les connaissances innées et peut ensuite être modifiée par des apprentissages. Ainsi, il a été prouvé que les ruminants, tout comme de nombreux autres animaux, sont capables d'associer les caractéristiques sensorielles des aliments à leurs conséquences post-ingestives au cours de processus d'apprentissage (Provenza 1995, Forbes et Provenza 2000). De tels apprentissages sont possibles aussi bien lorsque les conséquences associées aux caractéristiques sensorielles des aliments sont négatives (Zahoric et al 1990) que positives (Villalba et Provenza 1997,
Duncan et Young 2002), dès lors que la procédure de conditionnement permet à l'animal d'associer facilement une caractéristique sensorielle à une conséquence post-ingestive (cf. encadré). La palatabilité des aliments est également influencée par le fond génétique de l'animal, par son état interne - en référence à la notion d'alliesthésie (Brondel et Cabanac 2007) - et par l'environnement dans lequel il évolue (Forbes 2010). Par conséquent, le concept de palatabilité intègre plusieurs composantes : $i$ ) une palatabilité intrinsèque ou pré-conditionnée faisant référence à la valeur innée, positive ou négative, attribuée aux caractéristiques sensorielles des aliments au cours de l'évolution et/ou de pré-conditionnements in utero, ii) une palatabilité apprise ou conditionnée qui correspond à une réponse de l'animal aux aliments, basée sur des associations antérieures entre les caractéristiques sensorielles de l'aliment et ses conséquences post-ingestives et iii) une palatabilité reconditionnée liée au changement d'état de l'animal (stade physiologique, état de jeûne) ou à certains facteurs environnementaux tels que la température (Kissileff 1990, Forbes 2010). Etant donné qu'il existe de nombreuses difficultés méthodologiques à séparer les différentes composantes du concept général de palatabilité, nous utiliserons ici le terme «palatabilité » en référence à la capacité d'un aliment à provoquer une sensation agréable

\footnotetext{
${ }^{1}$ Ce texte est adapté d'une publication en langue anglaise dans la revue Animal (Favreau et al 2012).
} 
Encadré. Les apprentissages alimentaires : processus et méthodologie.

\section{Processus}

\begin{tabular}{|c|c|c|}
\hline $\begin{array}{c}\text { Caractéristiquessensorielles de } \\
\text { l'aliment } \\
\text { (forme, odeur, couleur, } \\
\text { texture, goût...) } \\
\text { Niveau pré-ingestif }\end{array}$ & $\begin{array}{c}\text { Conséquences digestiveset } \\
\text { métaboliques perçues } \\
\text { (récompensenutritionnelle, } \\
\text { encombrement, malaise...) } \\
\text { Niveau post-ingestif }\end{array}$ \\
Sélection ou évitementde l'aliment \\
$\begin{array}{c}\text { Réponse } \\
-y^{-}\end{array}$
\end{tabular}

- L'apprentissage alimentaire permet à l'animal d'associer les caractéristiques de l'aliment perçues avant la déglutition (niveau pré-ingestif) aux conséquences perçues après la déglutition (niveau post-ingestif), et d'adapter sa réponse vis-à-vis de cet aliment.

- Au niveau pré-ingestif, les caractéristiques sensorielles sont toutes les caractéristiques qui permettent à l'animal d'identifier un aliment via ses sens. Les conséquences post-ingestives peuvent être positives (e.g. récompense nutritionnelle, rectification d'un déséquilibre...) et/ou négatives (e.g. inconfort, douleur...).

- Une fois l'apprentissage établi, l'animal est capable de sélectionner l'aliment induisant des conséquences positives et d'éviter celui induisant des conséquences négatives.

\section{Méthodologie}

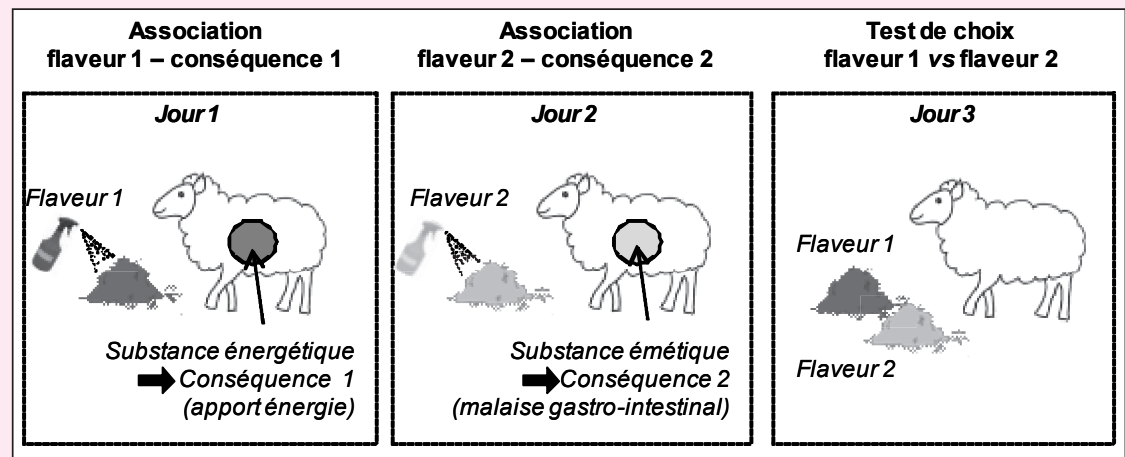

- Pour mettre en évidence un apprentissage alimentaire, on crée expérimentalement des associations " aliment - conséquence ». On utilise au minimum deux aliments que l'animal doit être capable de discriminer d'un point de vue sensoriel, par leurs flaveurs par exemple, mais identiques par ailleurs.

- Pendant l'ingestion de ces aliments, l'animal reçoit une conséquence post-ingestive qui peut être positive, par exemple du glucose pour un apport d'énergie, ou négative, par exemple une substance émétique pour induire un malaise gastro-intestinal. Chaque aliment est associé avec une seule conséquence.

- Le conditionnement se déroule sur plusieurs jours, la situation la plus simple consistant à proposer, chaque jour, une association " aliment-conséquence » différente. L'animal est ensuite soumis à un test de choix entre les deux aliments. La comparaison des réponses à ce test de choix à celles obtenues lors d'un test de choix entre les aliments avant le conditionnement permet de déterminer s'il y a eu un apprentissage.

- Dans le cas présenté dans cet encadré, on s'attend à ce que l'animal sélectionne l'aliment avec la flaveur 1 et évite celui avec la flaveur 2 . De nombreux résultats expérimentaux valident cette hypothèse.

immédiate, indépendamment des conséquences post-ingestives à venir et des facteurs environnementaux, mais avec un effet probable de l'état interne de l'animal, de son fond génétique et de ses apprentissages antérieurs.

Pour certains auteurs, le concept de palatabilité est très étroitement lié aux conséquences post-ingestives (Provenza 1995) voire même totalement déterminé par ces dernières (Garcia 1989). Elles motivation à consommer un aliment peut être provoquée uniquement par ses caractéristiques sensorielles attractives et alors passer outre les signaux postingestifs (Baumont et al 1990a, Ginane et al 2002). De plus, les caractéristiques sensorielles peuvent induire des préférences alimentaires immédiates, en l'absence de conséquences post-ingestives comme dans le cas du goût umami (Gherardi et Black 1991, Favreau et al 2010a). Les ruminants montrent également des difficultés à effectuer des apprentissages alimentaires lorsque le contexte d'alimentation est complexe. Tout ceci nous laisse donc supposer que le rôle des caractéristiques sensorielles des aliments dans le comportement alimentaire des ruminants domestiques a été sous-estimé jusqu'à présent et qu'il ne se limite pas au rôle d'agent discriminant. Par conséquent, cette synthèse a pour objectif de mettre en lumière les autres rôles, souvent méconnus, des caractéristiques sensorielles des aliments dans le comportement alimentaire des ruminants domestiques.

\section{1 / Discriminer différents items alimentaires}

L'unique rôle attribué aux caractéristiques sensorielles des aliments de manière consensuelle est celui qui permet aux animaux d'avoir connaissance de leur environnement alimentaire en discriminant différents items alimentaires par l'utilisation de leurs sens, seuls ou en les combinant. Les animaux utilisent un sens ou un autre en fonction de la distance qui les sépare de la ressource alimentaire. La vue et l'odorat aident l'animal à effectuer une première évaluation sensorielle de l'aliment sans aucun contact direct. Le toucher et le goût sont utilisés par l'animal dès lors qu'il entre en contact avec un aliment, au niveau de ses lèvres et/ou de sa bouche. L'odeur et le goût sont cependant souvent confondus lors d'une stimulation plus globale de la zone oropharyngée ; on parle alors de flaveur de l'aliment.

La vue est supposée être principalement utilisée dans le but d'orienter les herbivores au pâturage via un processus de discrimination à large échelle (Arnold 1966a). Elle peut également permettre aux animaux de discriminer des objets selon leur brillance (Bazely et Ensor 1989), des espèces végétales comme le trèfle et le ray-grass (Edwards et al 1997) et des polymorphismes de trèfle en se basant sur les marques blanches visibles sur leurs feuilles (Cahn et Harper 1976). Arnold (1966a) a également démontré que les régimes alimentaires sélectionnés par des moutons 
rendus temporairement aveugles sont de plus faible qualité nutritionnelle que ceux des moutons témoins. Dans ce cas, la vue est utilisée par les animaux pour discriminer et identifier des aliments, affectant ainsi le régime alimentaire sélectionné.

Le toucher joue également un rôle important puisque les herbivores " grazers » (consommateurs d'herbacées), tels que les ovins et les bovins, ne sélectionnent généralement pas les plantes rêches, dures, collantes ou épineuses (Baumont 1996, Vallentine 2001). A l'inverse, les chèvres qui sont des herbivores «browsers » (consommateurs d'herbacées et de ligneux), peuvent passer outre les défenses physiques de certains arbres car elles possèdent des zones dures dans leur bouche qui les insensibilisent aux effets des épines et des aiguillons (Cheeke et Dierenfield 2010). Krueger et al (1974) ont montré, chez les ovins, que le toucher est d'abord utilisé dans la sélection des espèces végétales pour compléter les informations recueillies par le goût, mais que les plantes sélectionnées, au moins en partie sur la base du toucher, appartiennent toutes à une catégorie de plantes de faible rugosité telles que la renouée (Polygonum montanum) ou encore l'oseille paucifolliée (Rumex paucifolium). Les caractéristiques physiques des plantes et le sens du toucher jouent aussi un rôle dans l'acceptabilité des variétés de brome chez le mouton (Arnold 1966b).

Le goût est le dernier sens utilisé par l'animal juste avant d'avaler l'aliment. Les cinq goûts primaires sont le sucré, le salé, l'amer, l'acide, auxquels s'est ajouté plus récemment le goût umami (Lindemann 2001). Diverses méthodes faisant appel au comportement animal ont été utilisées pour démontrer que les ruminants perçoivent et discriminent les cinq goûts primaires (Ginane et al 2011). Par exemple, des tests de choix entre deux bouteilles contenant des liquides différemment aromatisés (tests de type "two-bottle ») ont montré que les ovins, caprins et bovins sont sensibles aux goûts sucré, salé, amer et acide (Goatcher et Church 1970c, Krueger et al 1974). Ces sensibilités, évaluées via la plus faible concentration discriminée par les animaux, diffèrent aussi bien entre les espèces (les bovins sont plus sensibles au goût sucré que les caprins et ces derniers sont plus sensibles que les ovins) qu'entre les goûts, la sensibilité des ruminants allant en décroissant de l'amer, à l'acide, au salé et enfin au sucré (Goatcher et Church 1970a, Goatcher et Church 1970c). La sensibilité au goût amer varie en fonction du régime alimentaire des ruminants : les herbivores « grazers » consomment exclusivement des plantes herbacées et sont plus sensibles au goût amer que les herbivores "browsers » qui consomment aussi bien des plantes herbacées que des arbustes et des arbres (Glendinning 1994). Cet auteur a émis l'hypothèse que les herbivores "browsers" auraient évolué pour être moins sensibles au goût amer du fait qu'ils rencontrent fréquemment des plantes toxiques, présentant un goût amer, dans leur régime alimentaire.

Le goût umami, induit par le glutamate de sodium, est perçu par les ovins puisqu'il entraîne une préférence pour le fourrage auquel il est ajouté (Gherardi et Black 1991) et une augmentation de l'ingestion même lorsque les aliments sont expérimentalement dérivés du tube digestif (Colucci et Grovum 1993). De récentes études ont confirmé la perception du goût umami par les ruminants puisqu'elles ont montré que des agnelles augmentent leur préférence pour un aliment porteur du goût umami (Favreau et al 2010a, Villalba et al 2011, Bach et al 2012).

L'odorat est supposé avoir une moindre importance dans la sélection alimentaire que le goût (Krueger et al 1974), mais il est particulièrement difficile de différencier l'appréciation que font les animaux d'une odeur (provenant des composés volatils de l'aliment) par rapport à celle d'un goût (provenant des composés solubles de l'aliment). En effet, odeur et goût sont souvent couplés pour former une flaveur unique dès lors que l'aliment entre en bouche (Forbes et Mayes 2002). Des flaveurs naturelles ou artificielles sont fréquemment utilisées dans le cadre d'expériences de conditionnement; elles sont ajoutées à un aliment commun afin de créer deux aliments distincts d'un point de vue sensoriel mais identiques du point de vue des conséquences post-ingestives (Provenza et al 1996, Favreau et al 2010b). De nombreuses études ont ainsi démontré que les ovins discriminent différentes flaveurs artificielles telles que l'orange et l'anis (Kyriazakis et al 1997), l'oignon et la noix de coco (Villalba et al 1999) ou encore l'oignon utilisé à différentes concentrations (Villalba et Provenza 2000). Les ovins et les caprins discriminent également un aliment porteur d'une flaveur (érable, fraise, oignon...) du même aliment non traité (Robertson et al 2006). De rares études ont utilisé des odeurs naturelles comme des extraits aromatiques ou des composés naturels de plantes (acides tanniques, huile de bois de cèdre...) afin de démontrer que les ruminants sont capables de les discriminer, soit en comparant les ingestions de moutons normaux à celles de moutons anosmiques (Arnold et al 1980), soit en soumettant des chèvres à des tests de type cafétéria (Rosa et al 2002).

\section{2 / Procurer du plaisir ali- mentaire}

Du point de vue des neurosciences, la palatabilité se réfère à la composante hédonique de la récompense, le "liking », qui est responsable des réponses affectives aux aliments et qui induit le plaisir alimentaire (Berridge 1996, Berridge et Kringelbach 2008).

\section{1 / Comment évaluer la palata- bilité des aliments ?}

Pour évaluer la valeur hédonique des caractéristiques sensorielles des aliments, il est nécessaire d'observer la réponse comportementale des animaux à celles-ci, en évitant ou en maîtrisant les effets des conséquences post-ingestives associées à l'aliment pendant la durée de l'observation. De plus, afin de se soustraire de la palatabilité reconditionnée, il est important de contrôler l'état de faim et le stade physiologique des animaux.

Cela est possible, $i$ ) en mesurant des préférences alimentaires et/ou des ingestions à court terme (pendant quelques minutes), en faisant l'hypothèse qu'un tel intervalle de temps est insuffisant pour que les animaux ressentent les conséquences post-ingestives des aliments (Favreau et al 2010c), ii) en utilisant un ingrédient capable de modifier les caractéristiques sensorielles d'un aliment sans en modifier ses conséquences post-ingestives, par exemple en ajoutant un édulcorant sucré tel que la saccharine (Hellekant et al 1994), iii) en offrant différents items alimentaires simultanément puisqu'il a été démontré qu'une telle situation augmente considérablement la difficulté pour un animal à identifier quel item alimentaire est responsable de quelle conséquence (Duncan et Young 2002, Favreau et al 2010b) et iv) en vérifiant, à l'aide d'une procédure simple de conditionnement (cf. encadré), que la substance ajoutée modifie les caractéristiques sensorielles de l'aliment sans induire de conséquences post-ingestives à la dose utilisée (Gherardi et Black 1991, Favreau et al 2010a).

Deux autres méthodes permettant de maîtriser les conséquences post-ingestives des aliments ingérés peuvent également être utilisées. La première méthode consiste en une dérivation de l'alimentation des animaux via une canule œsophagienne, ce qui permet une stimulation sensorielle de la zone oro-pharyngée lors de l'ingestion tout 
en évitant les effets des conséquences post-ingestives des aliments puisqu'ils n'atteignent pas le rumen (Grovum et Chapman 1988, Colucci et Grovum 1993). La seconde méthode consiste à contrôler aussi bien les stimulations sensorielles de la zone oro-pharyngée que les conséquences post-ingestives du régime alimentaire. Cela est possible lorsqu'un aliment est consommé oralement tandis qu'un autre aliment est introduit, dans des proportions équivalentes, directement dans le rumen de l'animal via une fistule (Greenhalgh et Reid 1971, Favreau et al 2010c). La palatabilité des deux aliments testés (A et B) peut alors être évaluée par la comparaison des comportements alimentaires observés dans deux situations opposées d'un point de vue sensoriel mais similaires d'un point de vue de la composition globale du régime alimentaire et donc des conséquences post-ingestives, c'est-à-dire lorsque A est consommé oralement et B introduit dans le rumen, et inversement (figure 1).

Le conditionnement opérant peut également être un bon moyen d'évaluer la palatabilité d'un aliment. Cela consiste à appliquer des renforcements positifs ou négatifs à l'animal suite à l'expression d'un comportement ciblé. L'animal est libre d'agir ou non afin d'obtenir une récompense ou d'éviter une punition. En ce qui nous concerne, c'est la quantité de comportements (nombre d'appuis sur un levier, par exemple) que l'animal est prêt à exprimer pour obtenir un aliment qui nous renseigne sur sa palatabilité. Ainsi, des moutons ont été entraînés à presser un panneau pour pouvoir recevoir une récompense alimentaire ce qui a permis à Hutson et van Mourik (1981) de montrer que le taux de réponse était plus fort avec le blé et l'orge par rapport au pois, à la luzerne, au lupin et à l'avoine. Les animaux peuvent aussi être entraînés à marcher une certaine distance pour obtenir un aliment de bonne qualité tandis qu'un aliment de faible qualité est disponible à volonté ; plus la distance parcourue est importante, plus l'aliment de bonne qualité est préféré (Dumont et al 1998). Cette méthode a également permis de montrer que la palatabilité d'un aliment est augmentée en cas de restriction alimentaire (Schütz et al 2006, Verbeek et al 2011).

\section{2 / La palatabilité des aliments affecte-t-elle l'ingestion ?}

En utilisant la méthode permettant de contrôler les conséquences post-ingestives de façon à ce qu'elles soient similaires entre les traitements, Greenhalgh et Reid (1971) avaient trouvé des quantités ingérées journalières similaires lorsqu'ils ont comparé deux fourrages de qualité moyenne à bonne, un foin de prairie et de l'herbe déshydratée respectivement (figure 1). Néanmoins, dans le cas où ils ont utilisé un fourrage de mauvaise qualité (paille d'orge) face à un fourrage de bonne qualité (herbe déshydratée), ils ont observé une différence nette d'ingestion en faveur de l'herbe alors même que la composition de la ration totale était identique. Plus récemment, Favreau et al (2010c) ont utilisé la même méthode, également avec deux fourrages de qualité moyenne à bonne, un foin de prairie permanente et un foin de luzerne respectivement, et ont montré que l'ingestion journalière de matière sèche des ovins ne varie pas (figure 1). Toutefois, lorsque ces mêmes fourrages avaient été proposés en libre choix aux animaux, les moutons ont exprimé une très forte préférence, de l'ordre de $80 \%$ de l'ingéré total, pour le foin de luzerne (Favreau et al 2011). Ces différences de réponse des animaux selon les types de fourrages utilisés peuvent s'expliquer par un contraste sensoriel qui serait insuffisant entre les fourrages de qualité moyenne à bonne pour pou- voir influencer l'ingestion journalière indépendamment des conséquences post-ingestives. En revanche, quand ce contraste sensoriel est plus marqué, comme avec la paille d'orge et l'herbe déshydratée, il apparaît que les caractéristiques sensorielles peuvent influencer l'ingestion journalière indépendamment des conséquences post-ingestives. On ne peut toutefois pas exclure que les animaux aient mémorisé la faible valeur nutritive de la paille lors d'un apprentissage antérieur.

Les caractéristiques sensorielles des aliments peuvent également influencer l'ingestion des ruminants à l'échelle des repas. Il a été démontré que des ovins rassasiés effectuent un repas substantiel après une nouvelle distribution alimentaire, lorsque le fourrage offert est suffisamment palatable ; ce nouveau repas induit alors une augmentation de l'encombrement du rumen de plus de $10 \%$ par rapport au maximum journalier observé sans ce repas supplémentaire (Baumont et al 1990b). Les stimulations

Figure 1. Ingestions journalières (moy. \pm e.s. $g$ MS) pour différents fourrages à même conséquences post-ingestives (d'après Greenhalgh et Reid 1971, Favreau et al 2010c).

Cette méthode consiste à offrir à volonté un aliment à l'auge afin qu'il soit consommé oralement (aliment indiqué sur la partie supérieure de chaque barre d'histogramme) tandis qu'un autre aliment est introduit, en quantité équivalente, directement dans le rumen de l'animal via une fistule (aliment indiqué sur la partie inférieure de chaque barre d'histogramme). Ainsi, la valeur nutritive et les conséquences postingestives de la ration totale (aliment ingéré plus aliment introduit dans le rumen) sont identiques dans les deux traitements de chacune des expériences.

La première expérience (Greenhalgh et Reid 1971) utilise un fourrage de bonne qualité (herbe déshydratée, Hd, MAT = $139 \mathrm{~g} / \mathrm{kg} \mathrm{MS}$ ) et un fourrage de faible qualité (paille d'orge, Po, MAT = $39 \mathrm{~g} / \mathrm{kg} \mathrm{MS}$ ). Les deuxième (Greenhalgh et Reid 1971) et troisième expériences (Favreau et al 2010c) comparent des fourrages de qualité moyenne (respectivement : foin de prairie, Fp, MAT = $77 \mathrm{~g} / \mathrm{kg} \mathrm{MS}$ et foin de graminées, $\mathrm{Fg}, \mathrm{MAT}=72 \mathrm{~g} / \mathrm{kg} \mathrm{MS}$ ) à des fourrages de bonne qualité (respectivement : herbe déshydratée, Hd, MAT = $139 \mathrm{~g} / \mathrm{kg}$ MS et foin de luzerne, Luz, MAT = $156 \mathrm{~g} / \mathrm{kg}$ MS).

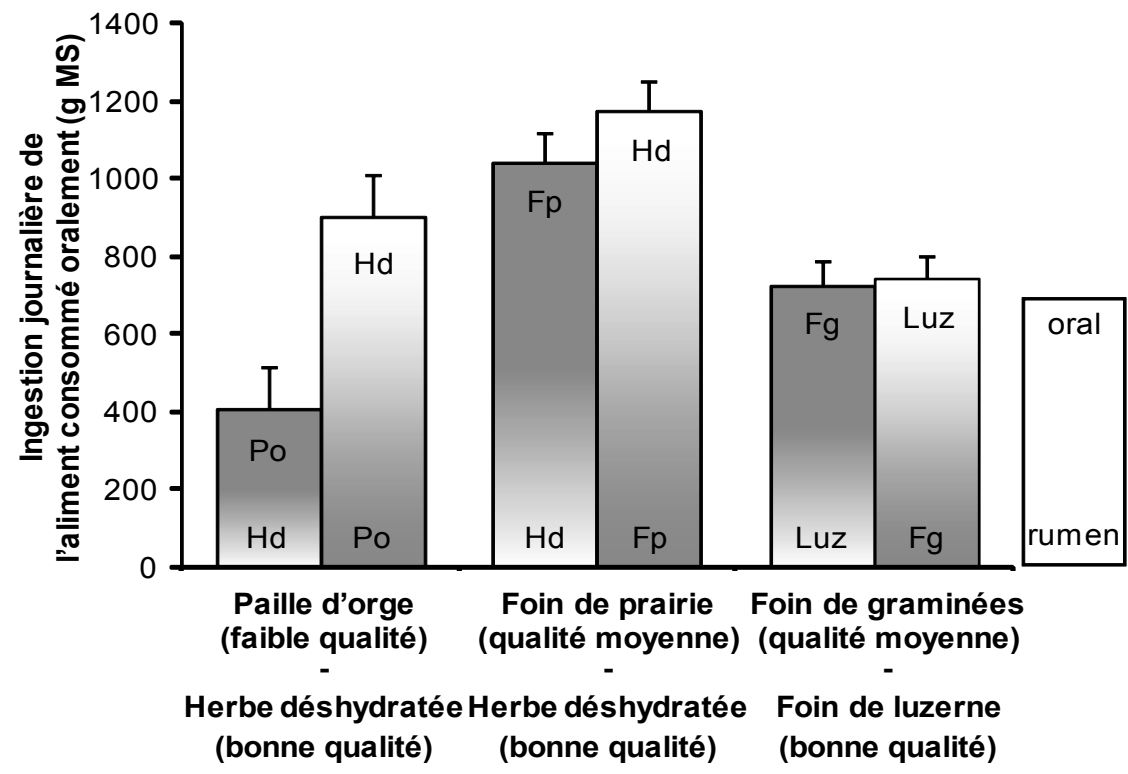


hédoniques induites par la nouvelle distribution surpassent ainsi les signaux de satiété liés à l'encombrement digestif. De la même manière, chez l'Homme, des apports modérés d'énergie avant le repas, combinés à des manipulations de la palatabilité au cours du repas (par l'assaisonnement des aliments), engendrent une surconsommation démontrant ainsi que la valeur hédonique des caractéristiques sensorielles des aliments diminue la capacité des indices de satiété à stopper le repas (Yeomans et al 2001). Chez les ovins, lorsque les conséquences postingestives sont contrôlées et similaires quel que soit le fourrage consommé oralement, l'ingestion d'un foin de luzerne pendant les premières minutes suivant la distribution est supérieure à celle d'un foin de prairie permanente, indiquant ainsi que la motivation à ingérer la luzerne est plus forte que les feedbacks négatifs liés à l'encombrement du rumen (Favreau et al 2010c). Enfin, l'ajout de glutamate de sodium (responsable du goût umami) augmente la palatabilité de la paille avec pour conséquence d'augmenter, à court terme (30 $\mathrm{min})$, la « fausse » ingestion de cet aliment chez des ovins équipés de dérivations œsophagiennes (Colucci et Grovum 1993). A l'inverse, une faible palatabilité peut entraîner une diminution de courte durée (10 min) de l'ingestion d'un co-produit des noix de palme, chez les bovins (Spörndly et Åsberg 2006).

\section{3 / La palatabilité des aliments affecte-t-elle les préférences ali- mentaires des ruminants?}

Certains résultats suggèrent que la valeur hédonique relative des caractéristiques sensorielles des aliments est capable d'induire des préférences alimentaires par elle-même, sans influence immédiate d'un quelconque renforcement post-ingestif, comme cela a été démontré dans le cas du goût umami (Grovum et Chapman 1988, Gherardi et Black 1991). Le goût umami semble avoir une valeur hédonique positive chez les ovins puisqu'il entraîne une préférence marquée et constante pour l'aliment additionné de glutamate de sodium quelles que soient les doses testées en comparaison du même aliment non traité (Gherardi et Black 1991, Favreau et al 2010a). Chez les bovins, une étude a montré que le goût umami engendre une préférence temporaire (de 3 à 6 semaines d'âge) chez des jeunes sevrés à l'âge de 3 semaines et testés entre 1 et 12 semaines d'âge (Waldern et van Dyk 1971). De la même manière, quand différentes flaveurs sont vaporisées sur un même aliment (i.e. pour assurer des conséquences post-ingestives similaires) et comparées les unes aux autres lors de tests de choix de courte durée, les animaux expriment souvent des préférences claires pour l'une ou l'autre des flaveurs. Des ovins ont effectivement préféré à $80 \%$ un foin de dactyle aromatisé à l'anis comparé à ce même foin de dactyle aromatisé à l'orange (Favreau et al 2010b), suggérant ainsi que la flaveur anis possède une valeur hédonique supérieure à celle de la flaveur orange chez les ovins. Ce résultat est cependant contradictoire avec ceux trouvés dans d'autres études dans lesquelles les ovins ne présentaient aucune préférence, dans un sens ou dans l'autre, pour ces deux flaveurs (Kyriazakis et al 1997, Arsenos et al 2000). Une telle variabilité est fréquemment rapportée dans la littérature à propos du goût sucré (voir la synthèse de Ginane et al 2011). La variabilité individuelle des préférences alimentaires entre différentes flaveurs ou différents goûts se rencontre dans de nombreux travaux (e.g. Goatcher et Church 1970a et 1970b, Robertson et al 2006). Elle peut être due aux effectifs animaux généralement limités dans les expériences et qui ne permettraient pas de rendre compte de la variabilité de la population totale. En l'absence de tout effet immédiat des conséquences post-ingestives des aliments, il apparaît logique de rencontrer une importante variabilité individuelle des préférences alimentaires, d'autant plus que les caractéristiques individuelles telles que l'état physiologique (Brondel et Cabanac 2007) et émotionnel (Tamashiro et al 2007) de l'animal et les expériences individuelles antérieures (Provenza 1995), in utero (Simitzis et al 2008) ou précoces (Miller-Cushon et DeVries 2010), sont reconnues comme étant capables d'influencer la palatabilité des aliments. Les expériences in utero et dans le jeune âge entraînent des modifications neurologiques, morphologiques et physiologiques du fait de l'interaction entre le génome de l'animal, son environnement et les conséquences postingestives des aliments ressenties; elles contribuent ainsi à la singularité de chaque individu (Provenza et al 2003).

\section{4 / La valeur hédonique de la diversité alimentaire}

Lorsque des ruminants ont un choix à faire entre des aliments ayant été consommés depuis plus ou moins longtemps, ils préfèrent généralement l'aliment qui n'a pas été consommé récemment. Cela est vrai pour des moutons qui préfèrent la première flaveur présentée lors d'une procédure de conditionnement simple par rapport à celle proposée en dernier (Arsenos et al 2000), pour des brebis qui préfèrent l'espèce végétale (trèfle blanc ou ray-grass) qui n'est pas celle qu'elles ont précédemment broutée (Newman et al 1992, Parsons et al 1994) et pour des génisses qui augmentent temporairement leur préférence pour le foin qui n'avait pas été offert au cours de la période expérimentale précédente (Ginane et al 2002). Ces auteurs ont suggéré que cette préférence pour l'aliment « nouveau » pourrait s'expliquer i) par la nécessité pour l'animal de sélectionner un régime alimentaire équilibré ou ii) parce que l'animal recherche une certaine rareté alimentaire pour des raisons fonctionnelles comme, par exemple, le maintien de la diversité de sa flore intestinale, ou encore iii) par un effet attractif de la nouveauté, considérée alors comme la recherche de diversité par l'animal. En effet, Scott et Provenza (1998) ont démontré que des agnelles préfèrent une diversité de flaveurs à une certaine monotonie lorsque le contenu nutritif des différents aliments proposés est maintenu constant. De la même manière, des moutons ont toujours préféré, à court terme, le foin qu'ils n'avaient pas l'habitude de consommer, quelle que soit sa nature (foin de luzerne ou foin de prairie permanente) et alors même que les conséquences post-ingestives associées aux différents régimes alimentaires étaient similaires (Favreau et al 2010c). Cette préférence pour la diversité sensorielle des aliments confirme que les caractéristiques sensorielles des aliments possèdent bien une dimension hédonique. Cela est en accord avec le concept de satiété sensorielle spécifique qui postule que la valeur hédonique des caractéristiques sensorielles d'un aliment décroît au fur et à mesure de sa consommation (Rolls 1986). Cela est également en accord avec des découvertes plus récentes qui montrent que l'état de bien-être des agnelles, estimé par le taux de cortisol une heure après le repas, est moindre avec un régime alimentaire monotone qu'avec un régime alimentaire diversifié (Villalba et al 2012). Les ruminants semblent ainsi rechercher la diversité alimentaire aussi bien pour satisfaire leurs besoins physiologiques que pour leur plaisir, ce qui pourrait avoir des implications sur le bien-être, par exemple pour des animaux nourris à l'auge avec une même ration pendant plusieurs semaines ou plusieurs mois, ou pour des animaux pâturant des couverts monospécifiques.

\section{5 / La palatabilité des aliments affecte-t-elle les apprentissages alimentaires ?}

Comme mentionné précédemment, des moutons ont montré une préférence initiale pour un foin de dactyle aromatisé à l'anis par rapport à ce même foin aromatisé à l'orange (Favreau et al 2010b). Après que ces mêmes moutons ont subi une procédure de conditionnement simple, leur préférence pour le foin associé à des conséquences post-ingestives négatives était toujours influencée par leurs préférences initiales pour les deux flaveurs. Ainsi, l'écart de préféren- 
ces entre les flaveurs observé avant les conditionnements négatifs s'est maintenu après (figure 2). Des résultats semblables ont été trouvés dans le cas d'une préférence initiale supérieure pour la flaveur fenugrec comparée à la flaveur d'ail (Arsenos et al 2000). Par conséquent, il apparaît que la palatabilité initiale d'un aliment influence son évaluation par l'animal dans un contexte d'apprentissage alimentaire, et que cela affecte la propension de l'animal à effectuer un apprentissage associatif entre caractéristiques sensorielles et conséquences post-ingestives.

\section{3 / Indiquer la valeur des conséquences post-ingesti- ves associées à un aliment}

La condition pour qu'une caractéristique sensorielle d'un aliment devienne un signal informatif est que les animaux soient capables de lui donner un sens via un processus évolutif et/ou via des apprentissages individuels. Quand les animaux se trouvent face à un nouvel aliment sans avoir aucune connaissance à son sujet, ils hésitent le plus généralement et vont même parfois jusqu'à refuser complètement de le consommer. Cette réponse néophobique est considérée comme adaptative car elle permet aux animaux d'éviter de consommer en trop grande quantité des aliments potentiellement dangereux lors de leur première rencontre et elle leur donne l'opportunité d'acquérir des connaissances à propos des conséquences postingestives associées à ces aliments inconnus (Birch 1999).

\section{1 / Les apprentissages alimen- taires et leurs limites}

Les ovins (du Toit et al 1991, Burritt et Provenza 1992), les bovins (Ralphs et Provenza 1999) et les caprins (Duncan et Young 2002) sont capables d'associer les caractéristiques sensorielles des ali-

Figure 2. Préférence (moy. \pm e.s.) pour une flaveur associée à une conséquence post-ingestive négative, avant et après conditionnements (d'après Favreau et al 2010b).

Pour le premier lot de moutons, le foin de dactyle aromatisé à l'anis était associé à une conséquence post-ingestive négative alors que ce même fourrage aromatisé à l'orange était associé à une conséquence positive. Pour le second lot de moutons, la situation était inversée (la flaveur orange était associée à une conséquence négative tandis que la flaveur anis était associée à une conséquence positive). Le graphique présente les préférences des animaux pour ces deux flaveurs, mesurées lors de tests de choix ayant été réalisés avant tout conditionnement, après une première puis une seconde période de conditionnement. L'écart de préférences entre les flaveurs, observé avant tout conditionnement, s'est maintenu après deux périodes de conditionnements négatifs.

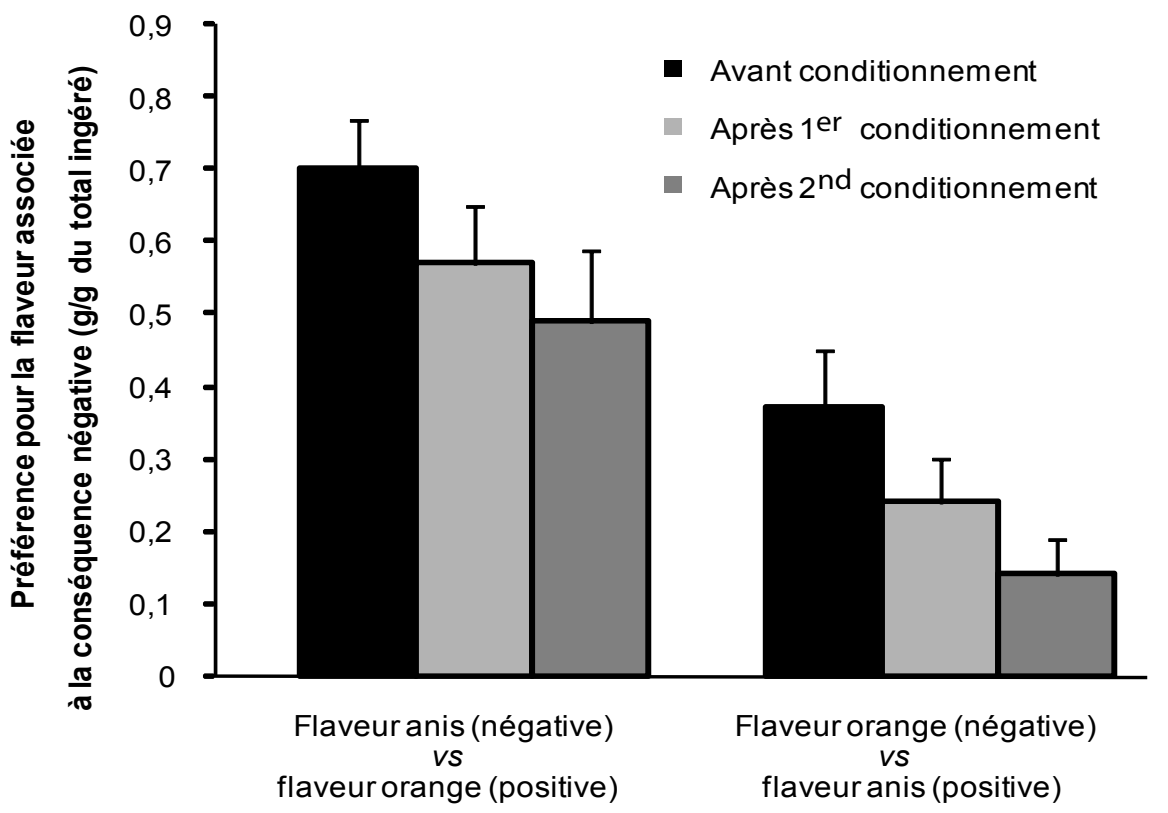

\footnotetext{
2 L'aposématisme (Lindström 1999) est une stratégie adaptative qui se traduit par le fait que certains animaux émettent des signaux d'avertissement clairement perceptibles pouvant être visuels (le plus souvent il s'agit d'une coloration d'avertissement), sonores ou chimiques. On suppose que ces signaux aposématiques représentent un moyen de défense puisqu'ils avertissent les prédateurs éventuels que les animaux qui les portent représentent un danger et qu'ils doivent donc éviter de les consommer.
} 
expriment une aversion pour un aliment familier quand un indicateur olfactif préalablement associé à une toxicité lui était ajouté (Provenza et al 2000) ; ceci est vrai aussi bien dans le cas où l'indicateur est une flaveur que l'animal n'a pas l'occasion de rencontrer dans son milieu naturel (flaveur « artificielle » noix de coco) que dans le cas où c'est une odeur naturelle (l'odeur d'Astragalus bisulcatus). Dans cette étude, il apparaît donc que les caractéristiques sensorielles de l'aliment ont acquis un sens, indiquant une éventuelle toxicité aux animaux, ce qui, par conséquent, réduit leur préférence pour l'aliment associé à cet indicateur. A l'inverse, les caractéristiques sensorielles peuvent indiquer une conséquence post-ingestive positive. En effet, une néophobie alimentaire a pu être évitée en additionnant une flaveur ou une odeur familière à un aliment nouveau (Launchbaugh et al 1997, Van Tien et al 1999). La familiarité a pu alors indiquer à l'animal que l'aliment est sans danger (Buritt et Provenza 1989).
L'utilisation de flaveurs que les animaux avaient associées préalablement à des conséquences post-ingestives positives ou négatives, a permis à des ovins d'améliorer leur capacité d'apprentissage alimentaire dans un environnement alimentaire complexe par rapport à des moutons naïfs qui ne bénéficiaient pas de ce premier apprentissage (Favreau et al 2010b). Ce résultat montre que les moutons peuvent attribuer un rôle d'indicateur de la valeur post-ingestive des aliments à une caractéristique sensorielle et généraliser cette connaissance d'une flaveur à différentes situations qui varient en complexité. Ainsi, les ovins peuvent se reposer sur des indicateurs sensoriels pour obtenir une aide essentielle dans leurs apprentissages alimentaires, et d'autant plus quand l'environnement d'apprentissage est complexe. Cela confirme des résultats antérieurs concernant cette capacité de généralisation (Launchbaugh et Provenza 1994, Ginane et Dumont 2006). Howery et al (2000) ont démontré que des bovins ont également été capables d'associer des indices visuels (des barrières et des cônes de signalisation) à des aliments dont la qualité varie, et qu'ils ont utilisé ces informations pour rechercher des ressources alimentaires plus efficacement dans des environnements complexes dans lesquels la localisation des ressources alimentaires varie.

Les espèces végétales rencontrées au pâturage par les animaux diffèrent par la multiplicité et la diversité de leurs caractéristiques sensorielles et notamment par la variabilité de leurs profils gustatifs. Certains de ces profils pourraient révéler la valeur post-ingestive des aliments. En effet, de nombreux composés secondaires présents dans les espèces végétales peuvent induire des effets négatifs (réduction de digestibilité voire toxicité) et présentent souvent un goût amer (Garcia et Hankins 1975, Rogosic et al 2008). A l'inverse, les goûts sucrés et umami indiqueraient, respectivement, la présence d'énergie (Swithers et Davidson 2008) et de pro-

Figure 3. Rôles et influences des caractéristiques sensorielles et des conséquences post-ingestives des aliments sur le comportement alimentaire des ruminants domestiques.

Les conséquences post-ingestives des aliments jouent un rôle prépondérant dans le comportement alimentaire des ruminants (flèches noires). Elles contrôlent directement l'ingestion (par leurs effets d'encombrement du rumen et/ou leurs effets nutritifs/toxiques) et influencent indirectement l'ingestion et les préférences alimentaires via les apprentissages.

Les flèches rouges représentent les différents rôles des caractéristiques sensorielles des aliments :

1 - Toutes les caractéristiques sensorielles des aliments sont des agents discriminants qui aident les animaux à différencier les items alimentaires rencontrés, et facilitent ainsi leurs associations à des conséquences post-ingestives par apprentissage.

2- Certaines caractéristiques sensorielles possèdent, en plus, une valeur hédonique qui influence directement les préférences, l'ingestion, mais aussi le processus d'apprentissage.

3- Enfin, seulement certaines de ces caractéristiques sensorielles ont acquis une valeur positive ou négative via un apprentissage individuel ou un processus évolutif. Cette valeur peut alors jouer un rôle d'indicateur permettant d'aider les ruminants à anticiper les conséquences post-ingestives associées à l'aliment et d'améliorer leur capacité d'apprentissage alimentaire, en particulier dans des environnements complexes.

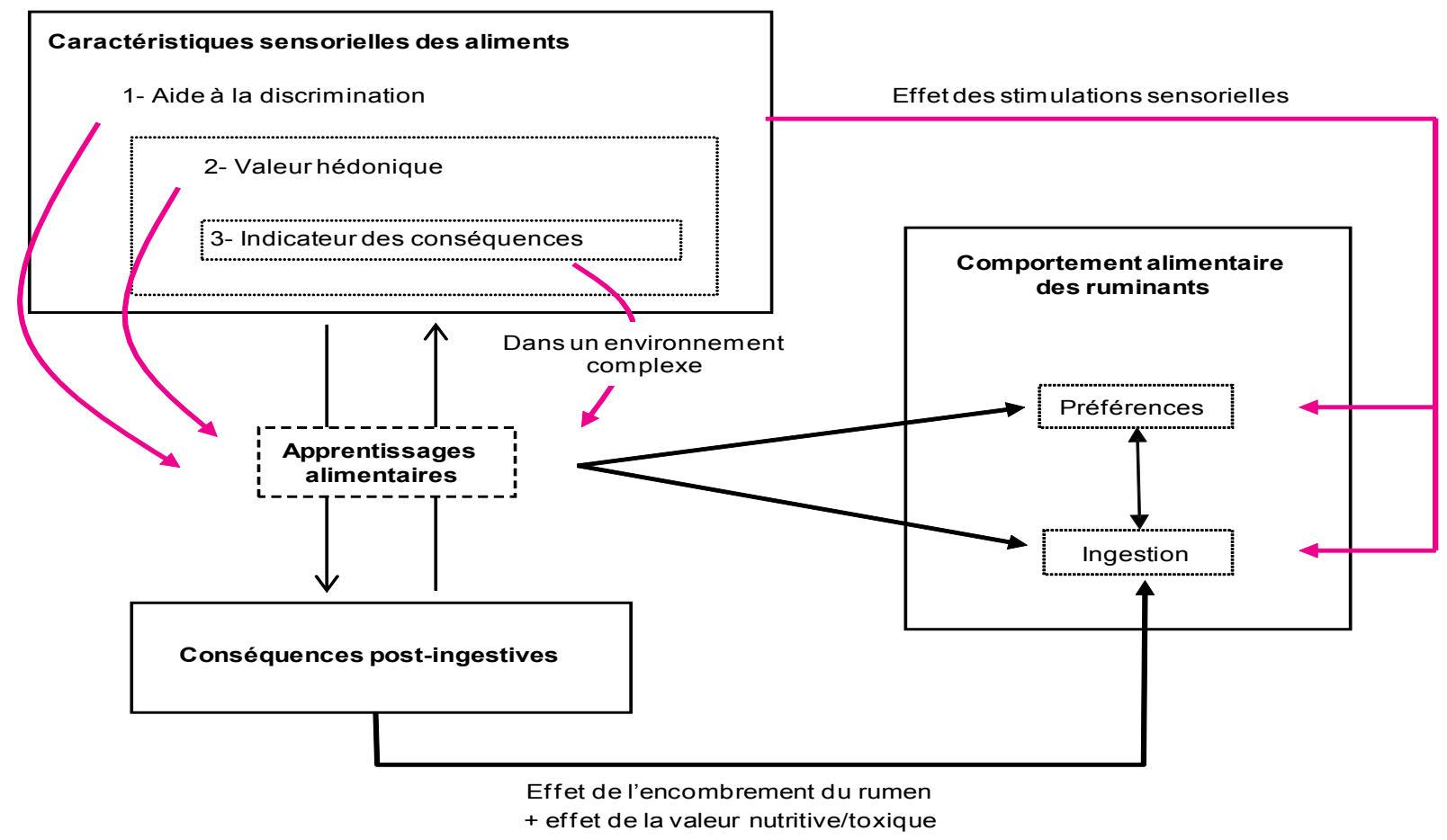


téines (Naim et al 1991) dans l'aliment. Des préférences initiales marquées pour le goût umami ont été observées dans différents contextes (Waldern et van Dyk 1971, Gherardi et Black 1991, Favreau et al 2010a) ce qui est en accord avec son rôle d'indicateur d'une valeur post-ingestive positive. De plus, une étude récente a observé les préférences d'agneaux, alimentés avec un régime déficient ou excédentaire en protéines, et auxquels était proposé le choix entre ce même régime avec ou sans le goût umami. Les résultats montrent clairement que les agneaux soumis à un déficit protéique ont une préférence pour le goût umami bien plus importante (environ $72 \%$ ) que ceux expérimentant la situation inverse $(39 \%)$ (Bach et al 2012). Le goût amer pourrait, quant à lui, posséder une valeur post-ingestive négative puisque des moutons ont montré une certaine réticence à augmenter leur préférence pour ce goût, même quand il était associé à des conséquences post-ingestives positives (Favreau et al 2010a). Il semblerait donc que les herbivores attribuent une valeur a priori aux différents goûts primaires. Par conséquent, les ruminants pourraient utiliser ces goûts primaires en tant qu'indicateurs afin d'anticiper les conséquences post-ingestives des aliments qu'ils rencontrent et alors effectuer une recherche de ressources alimentaires plus efficace et plus sûre leur permettant notamment d'éviter les aliments toxiques.

\section{Conclusion}

Cette synthèse met en lumière que les rôles des caractéristiques sensorielles des aliments avaient été précédemment sous-estimés ou simplifiés. Elle souligne les trois rôles majeurs des caractéristiques sensorielles des aliments dans le comportement alimentaire des ruminants (figure 3). Premièrement, toutes les caractéristiques sensorielles des aliments peuvent être considérées comme des agents discriminants qui permettent aux animaux de distinguer différents items alimentaires. Deuxièmement, certaines de ces caractéristiques sensorielles possèdent une valeur hédonique qui peut influencer l'ingestion, les préférences et les apprentissages alimentaires des ruminants, indépendamment de toute conséquence post-ingestive immédiate. Troisièmement, certaines de ces caractéristiques sensorielles ont acquis, par le biais de processus évolutifs ou d'apprentissages individuels antérieurs, un rôle d'indicateur de la valeur de l'aliment permettant à l'animal d'anticiper les conséquences postingestives et ainsi d'améliorer son efficacité à rechercher les aliments les plus intéressants et à effectuer de nouveaux apprentissages alimentaires. Ceci présente un atout certain pour les animaux vivant dans des environnements complexes.

Cette synthèse suggère également des pistes pour améliorer le bien-être des ruminants et leur production en prenant en compte les multiples rôles des caractéristiques sensorielles des aliments. Par exemple, des caractéristiques sensorielles peuvent être utilisées pour améliorer la palatabilité de fourrages pauvres ou d'aliments nouveaux afin d'augmenter leur acceptabilité par l'animal, au moins à court terme. De plus, les animaux semblent rechercher la diversité des caractéristiques sensorielles de leur alimentation. Enrichir l'environnement alimentaire des ruminants d'élevage pourrait être une manière relativement simple d'améliorer leur bien-être. Des indicateurs sensoriels pourraient également être utilisés par les éleveurs pour apprendre aux animaux à éviter les plantes toxiques ou au contraire empêcher les animaux de consommer des plantes que l'on souhaite préserver.

\section{Remerciements}

Les auteurs tiennent à remercier le département PHASE de l'INRA et la région Auvergne pour la bourse de thèse qu'ils ont accordée à Angélique Favreau pour ce projet de recherche.

\section{Références}

Arnold G.W., 1966a. The special senses in grazing animals I- Sight and dietary habits in sheep. Aust. J. Agric. Res., 17, 523-529.

Arnold G.W., 1966b. The special senses in grazing animals II- Smell, taste, and touch and dietary habits in sheep. Aust. J. Agric. Res., 17, 531-542.

Arnold G.W., De Boer E.S., Boundy C.A.P., 1980. The influence of odour and taste on the food preferences and food intake of sheep. Aust. J. Agric. Res., 31, 571-587.

Aronsson M., Gamberale-Stille G., 2008. Domestic chicks primarily attend to colour, not pattern, when learning an aposematic coloration. Anim. Behav., 75, 417-423.

Arsenos G., Hills J., Kyriazakis I., 2000. Conditioned feeding responses of sheep towards flavoured foods associated with casein administration: the role of long delay learning. Anim. Sci., 70, 157-169.

Bach A., Villalba J.J., Ipharraguerre I.R., 2012. Interactions between mild nutrient imbalance and taste preferences in young ruminants. J. Anim. Sci., 90, 1015-1025.

Baumont R.,1996. Palatability and feeding behaviour in ruminants. A review. Ann. Zootech., 45, 385-400.

Baumont R., Malbert C.H., Ruckebusch Y., 1990a. Mechanical stimulation of rumen fill and alimentary behaviour in sheep. Anim. Prod., 50, 123-128.

Baumont R., Seguier N., Dulphy J.P., 1990b. Rumen fill, forage palatability and alimentary behaviour in sheep. J. Agric. Sci., 115, 277-284.

Bazely D.R., Ensor C.V., 1989. Discrimination learning in sheep with cues varying in brightness and hue. Appl. Anim. Behav. Sci., 23, 293-299.

Berridge K.C., 1996. Food reward: Brain substrates of wanting and liking. Neurosci. Biobehav. Rev., 20, 1-25.

Berridge K.C., Kringelbach M., 2008. Affective neuroscience of pleasure: reward in humans and animals. Psychopharmacology, 199, 457-480

Birch L.L., 1999. Development of food preferences. Annu. Rev. Nutr., 19, 41-62.

Brondel L., Cabanac M., 2007. Alliesthesia in visual and auditory sensations from environmental signals. Physiol. Behav., 91, 196-201.

Burritt E.A., Provenza F.D., 1989. Food aversion learning: ability of lambs to distinguish safe from harmful foods. J. Anim. Sci., 67, 1732-1739.

Burritt E.A., Provenza F.D., 1992. Lambs form preferences for nonnutritive flavors paired with glucose. J. Anim. Sci., 70, 11331136.
Cahn M.G., Harper J.L., 1976. The biology of the leaf mark polymorphism in Trifolium repens L. Heredity, 37, 327-333.

Cheeke P.R., Dierenfeld E.S., 2010 Comparative animal nutrition and metabolism. CABI, Cambridge, Grande Bretagne, 352p.

Colucci P.E., Grovum W.L., 1993. Factors affecting the voluntary intake of food by sheep 6 . The effect of monosodium glutamate on the palatability of straw diets by sham-fed and normal animals. Br. J. Nutr., 69, 37-47.

du Toit J.T., Provenza F.D., Nastis A., 1991. Conditioned taste aversions: how sick must a ruminant get before it learns about toxicity in foods? Appl. Anim. Behav. Sci., 30, 35-46.

Dumont B., 1997. Diet preferences of herbivores at pasture. Ann. Zootech., 46, 105-116.

Dumont B., Dutronc A., Petit M., 1998. How readily will sheep walk for a preferred forage? J. Anim. Sci., 76, 965-971.

Duncan A.J., Young S.A., 2002. Can goats learn about foods through conditioned food aversions and preferences when multiple food options are simultaneously available? J. Anim. Sci., 80, 2091-2098.

Duncan A.J., Elwert C., Villalba J.J., Yearsley J., Pouloupoulou I., Gordon I.J., 2007. How does pattern of feeding and rate of nutrient delivery influence conditioned food preferences? Oecologia, 153, 617-624. 
Edwards G.R., Newman J.A., Parsons A.J., Krebs J.R., 1997. Use of cues by grazing animals to locate food patches: an example with sheep. Appl. Anim. Behav. Sci., 51, 59-68.

Favreau A., Baumont R., Ferreira G., Dumont B., Ginane C., 2010a. Do sheep use umami and bitter tastes as cues of post-ingestive consequences when selecting their diet? Appl. Anim. Behav. Sci., 125, 115-123.

Favreau A., Baumont R., Duncan A.J., Ginane C., 2010b. Sheep use preingestive cues as indicators of postingestive consequences to improve food learning. J. Anim. Sci., 88, 1535 1544.

Favreau A., Ginane C., Baumont R, 2010c. Feeding behaviour of sheep fed lucerne $v$ grass hays with controlled post-ingestive consequences. Animal, 4, 1368-1377.

Favreau A., Ginane C., Baumont R., 2011. The hedonic value of food diversity in sheep is not only a matter of choice. Adv. Anim. Biosci., 2, 344.

Favreau-Peigné A., Baumont R., Ginane C., 2012. Food sensory characteristics: their unconsidered roles in the feeding behaviour of domestic ruminants. Animal, doi:10.1017S1751731112002145.

Forbes J.M., 2010. Palatability: principles, methodology and practice for farm animals. CAB Reviews: Perspectives in Agriculture, Veterinary Science, Nutrition and Natural Resources, 5, 1-15.

Forbes J.M., Provenza F.D., 2000 Integration of learning and metabolic signals into a theory of dietary choice and food intake. In Ruminant Physiology: Digestion, Metabolism, Growth and Reproduction. Cronje P.B. (Ed). Department of Animal and Wildlife Science, University of Pretoria, Afrique du Sud, 3-19.

Forbes J.M., Mayes R.W., 2002. Food choice. In Sheep nutrition. Freer M., Dove H. (Eds). CSIRO Plant Industry, Canberra, Australie, 51-69.

Garcia J., 1989. Food for Tolman: coginition and cathexis in concert. In Aversion, avoidance, and anxiety: Perspectives on aversively motivated behavior. Archer T., Nilsson L.G. (Eds). Lawrence Erlbaum Associates, Hillsdale, $\mathrm{NJ}, 45-85$.

Garcia J., Hankins W.G., 1975. The evolution of bitter and the acquisition of toxiphobia. In Olfaction and Taste: Fifth International Symposium. Denton D., Coghlan J. (Eds). Academic Press, New York, Etats-Unis, 39-45.

Gherardi S.G., Black J.L., 1991. Effect of palatability on voluntary feed intake by sheep. I- Identification of chemicals that alter the palatability of a forage. Aust. J. Agric. Res., $42,571-584$

Ginane C., Dumont B., 2006. Generalization of conditioned food aversions in grazing sheep and its implication for food categorization. Behav. Process., 73, 178-186.

Ginane C., Baumont R., Lassalas J., Petit M., 2002. Feeding behaviour and intake of heifers fed on hays of various quality, offered alone or in a choice situation. Anim. Res., 51, 177-188.

Ginane C., Duncan A.J., Young S.A., Elston D.A., Gordon I.J., 2005. Herbivore diet selection in response to simulated variation in nutrient rewards and plant secondary compounds. Anim. Behav., 69, 541-550.
Ginane C., Baumont R., Favreau-Peigné A., 2011. Perception and hedonic value of basic tastes in domestic ruminants. Physiol. Behav., 104, 666-674.

Glendinning J.I., 1994. Is the bitter taste rejection always adaptive? Physiol. Behav., 56, 1217-1227.

Goatcher W.D., Church D.C., 1970a. Taste responses in ruminants. III. Reactions of pygmy goats, normal goats, sheep and cattle to sucrose and sodium chloride. J. Anim. Sci., 31, 364-372.

Goatcher W.D., Church D.C., 1970b. Taste responses in ruminants. IV. Reactions of pygmy goats, normal goats, sheep and cattle to acetic acid and quinine hydrochloride. J. Anim. Sci., 31, 373-382.

Goatcher W.D., Church D.C., 1970c. Review of some nutritional aspects of the sense of taste. J. Anim. Sci., 31, 973-981.

Greenhalgh J.F.D., Reid G.W., 1971 Relative palatability to sheep of straw, hay and dried grass. Br. J. Nutr., 26 107-116.

Grovum W.L., Chapman H.W., 1988. Factors affecting the voluntary intake of food by sheep. 4.The effect of additives representing the primary tastes on sham intakes by oesophageal-fistulated sheep. Br. J. Nutr., 59, 63-72

Hellekant G., Hård Af Segerstad C., Roberts T.W., 1994. Sweet taste in the calf: III. Behavioral responses to sweeteners. Physiol. Behav., 56, 555-562.

Howery L.D., Bailey D.W., Ruyle G.B., Renken W.J., 2000. Cattle use visual cues to track food locations. Appl. Anim. Behav. Sci., 67, 1-14.

Hutson G.D., Van Mourik S.C., 1981. Food preferences of sheep. Aust. J. Exp. Agric. Anim. Husb., 21, 575-582.

Kissileff H.R., 1990. Some suggestions on dealing with palatability - Response to Ramirez. Appetite, 14, 162-166.

Krueger W.C., Laycock W.A., Price D.A., 1974. Relationship of taste, smell, sight, and touch to forage selection. J. Range Manage. 27, 258-262.

Kühnle A., Müller C., 2011. Relevance of visual and olfactory cues for host location in the mustard leaf beetle Phaedon cochleariae. Physiol. Entomol., 36, 68-76.

Kyriazakis I., Papachristou T.G., Duncan A.J., Gordon I.J., 1997. Mild conditioned food aversions developed by sheep towards flavors associated with plant secondary compounds. J. Chem. Ecol., 23, 727-746.

Launchbaugh K.L., Provenza F.D., 1994. The effect of flavor concentration and toxin dose on the formation and generalization of flavor aversions in lambs. J. Anim. Sci., 72, 10-13.

Launchbaugh K.L., Provenza F.D., Werkmeister M.J., 1997. Overcoming food neophobia in domestic ruminants through addition of a familiar flavor and repeated exposure to novel foods. Appl. Anim. Behav. Sci., 54, 327334.

Lindström L., 1999. Experimental approaches to studying the initial evolution of conspicuous aposematic signalling. Evol. Ecol. 13, 605618

Lindemann B., 2001. Receptors and transduction in taste. Nature, 413, 219-225.
Miller-Cushon E.K., DeVries T.J., 2010. Effect of early feed type exposure on dietselection behavior of dairy calves. J. Dairy Sci., 94, 342-350.

Naim M., Ohara I., Kare M.R., Levinson M. 1991. Interaction of MSG taste with nutritionperspectives in consummatory behavior and digestion. Phyiol. Behav., 49, 1019-1024.

Newman J.A., Parsons A.J., Harvey A., 1992. Not all sheep prefer clover: diet selection revisited. J. Agric. Sci., 119, 275-283.

Parsons A.J., Newman J.A., Penning P.D. Harvey A., Orr R.J., 1994. Diet preference of sheep: effects of recent diet, physiological state and species abundance. J. Anim. Ecol., 63, 465-478.

Provenza F.D., 1995. Postingestive feedback as an elementary determinant of food preference and intake in ruminants. J. Range Manage., 48, 2-17.

Provenza F.D., Scott C.B., Phy T.S., Lynch J.J., 1996. Preference of sheep for foods varying in flavors and nutrients. J. Anim. Sci., 74, 2355-2361.

Provenza F.D., Kimball B.A., Villalba J.J., 2000. Roles of odor, taste, and toxicity in the food preferences of lambs: implications for mimicry in plants. Oikos, 88, 424-432.

Provenza F.D., Villalba J.J., Dziba L.E., Atwood S.B., Banner R.E., 2003. Linking herbivore experience, varied diets, and plant biochemical diversity. Small Rum. Res., 49, 257-274.

Ralphs M.H., Provenza F.D., 1999 Conditioned food aversions: principles and practices, with special reference to social facilitation. Proc. Nutr. Soc., 58, 813-820.

Robertson E., Gordon I.J., Pérez-Barbería F.J., 2006. Preferences of sheep and goats for straw pellets treated with different foodflavouring agents. Small Rum. Res., 63, 5055

Rogers P.J., 1990. Why a palatability construct is needed. Appetite, 14, 167-170.

Rogosic J., Estell R.E., Ivankovic S., Kezic J., Razov J., 2008. Potential mechanisms to increase shrub intake and performance of small ruminants in mediterranean shrubby ecosystems. Small Rum. Res., 74, 1-15.

Rolls B.J., 1986. Sensory-specific satiety. Nutr. Rev., 44, 93-101.

Rosa G.D., Moio L., Napolitano F., Grasso F., Gubitosi L., Bordi A., 2002. Influence of flavor on goat feeding preferences. J. Chem. Ecol., 28, 269-281.

Schütz K., Davison D., Matthews L., 2006. Do different levels of moderate feed deprivation in dairy cows affect feeding motivation? Appl. Anim. Behav. Sci., 101, 253-263.

Scott L.L., Provenza F.D., 1998. Variety of foods and flavors affects selection of foraging location by sheep. Appl. Anim. Behav. Sci., 61, 113-122.

Simitzis P.E., Bizelis J.A., Deligeorgis S.G., Feggeros K., 2008. Effect of early dietary experiences on the development of feeding preferences in semi-intensive sheep farming systems-a brief note. Appl. Anim. Behav. Sci., 111, 391-395.

Skelhorn J., Griksaitis D., Rowe C., 2008. Colour biases are more than a question of taste. Anim. Behav., 75, 827-835. 
Spörndly E., Åsberg T., 2006. Eating rate and preference of different concentrate components for cattle. J. Dairy Sci., 89, 2188-2199.

Swithers S.E., Davidson T.L., 2008. A role for sweet taste: calorie predictive relations in energy regulation by rats. Behav. Neurosci., $122,161-173$.

Tamashiro K.L.K., Hegeman M.A., Nguyen M.M.N., Melhorn S.J., Ma L.Y., Woods S.C., Sakai R.R., 2007. Dynamic body weight and body composition changes in response to subordination stress. Physiol. Behav., 91, 440448.

Vallentine J.F., 2001. Grazing Management. Academic Press, San Diego, Etats-Unis, 659p.

Van Tien D., Lynch J.J., Hinch G.N., Nolan J.V., 1999. Grass odor and flavor overcome feed neophobia in sheep. Small Rum. Res., 32, 223-229.

Verbeek E., Waas J.R., McLeay L., Matthews L.R., 2011. Measurement of feeding motivation in sheep and the effects of food restriction Appl. Anim. Behav. Sci., 132, 121-130.

Villalba J.J., Provenza F.D., 1997. Preference for wheat straw by lambs conditioned with intraruminal infusions of starch. Br. J. Nutr., 77 287-297.

Villalba J.J., Provenza F.D., 2000. Roles of flavor and reward intensities in acquisition and generalization of food preferences: do strong plant signals always deter herbivory? J. Chem. Ecol., 26, 1911-1922.

Villalba J.J., Provenza F.D., Rogosic J., 1999. Preference for flavored wheat straw by lambs conditioned with intraruminal infusions of starch administered at different times after straw ingestion. J. Anim. Sci., 77, 3185-3190.

Villalba J.J., Bach A., Ipharraguerre I.R. 2011. Feeding behaviour and performance of lambs are influenced by flavor diversity. J. Anim. Sci., 89, 2571-2581.
Villalba J.J., Catanese F., Provenza F.D. Distel R.A., 2012. Relationships between early experience to dietary diversity, acceptance of novel flavors, and open field behavior in sheep. Physiol. Behav., 105, 181-187.

Waldern D.E., van Dyk R.D., 1971. Effect of monosodium glutamate in starter rations on feed consumption and performance of early weaned calves. J. Dairy Sci., 54, 262-265.

Yeomans M.R., Lee M.D., Gray R.W., French S.J., 2001. Effects of test-meal palatability on compensatory eating following disguised fat and carbohydrate preloads. Int. J. Obesity, 25, 1215-1224

Zahorik D.M., Houpt K.A., SwartzmanAndert J., 1990. Taste-aversion learning in three species of ruminants. Appl. Anim. Behav. Sci., 26, 27-39.

\section{Résumé}

Pour évaluer les aliments et orienter leurs choix alimentaires, les ruminants utilisent les informations pré-ingestives (c'est-à-dire les caractéristiques sensorielles des aliments perçues avant leur déglutition) et les informations post-ingestives (c'est-à-dire les conséquences digestives et métaboliques expérimentées par les animaux après la déglutition). Des associations entre informations pré- et post-ingestives peuvent être apprises par les animaux, leur permettant ainsi d'éviter les aliments qui induisent des conséquences négatives et de sélectionner ceux qui induisent des conséquences positives. Les informations post-ingestives sont considérées comme étant le principal facteur influençant le comportement alimentaire des ruminants, alors que les caractéristiques sensorielles des aliments seraient utilisées uniquement pour discriminer les aliments et pour les associer aux conséquences post-ingestives lors de processus d'apprentissage. Cependant, les caractéristiques sensorielles jouent également d'autres rôles. Premièrement, certaines caractéristiques sensorielles possèdent une valeur hédonique qui influence l'ingestion, les préférences et l'apprentissage de la valeur des aliments indépendamment des conséquences post-ingestives immédiates. Deuxièmement, certaines caractéristiques sensorielles deviennent des indicateurs des conséquences post-ingestives après que leur valeur hédonique initiale a acquis une valeur positive ou négative via un apprentissage ou le processus évolutif. Ces caractéristiques jouent alors le rôle de signal permettant d'aider les ruminants à anticiper les conséquences post-ingestives et à améliorer leur capacité d'apprentissage des conséquences des aliments, en particulier dans des environnements alimentaires complexes. Les caractéristiques sensorielles des aliments pourraient être utilisées pour procurer un plaisir alimentaire aux animaux, augmenter la palatabilité des aliments et accroître la capacité d'apprentissage des animaux dans des situations alimentaires complexes, autant d'éléments de nature à améliorer leur bien-être et leur productivité.

\section{Abstract}

\section{The role of food sensory characteristics in the feeding behaviour of domestic ruminants}

When domestic ruminants are faced with food diversity, they can use pre-ingestive information (food sensory characteristics perceived by the animal before swallowing the food) and post-ingestive information (digestive and metabolic consequences experienced by the animal after swallowing the food) to evaluate the food and select a suitable diet. Pre- and post-ingestive information are associated in food learning processes, allowing the animal to avoid food that induces negative consequences while selecting those inducing positive consequences. Post-ingestive information is considered as the main force that influences feeding behaviour whereas food sensory characteristics are only used to discriminate between different foods and to be associated with post-ingestive information in learning processes. Food sensory characteristics can however play other roles. First, some sensory characteristics also possess a hedonic value which influences ruminants' intake, preferences and food learning independently of any immediate post-ingestive consequences. Second, some of these food sensory characteristics become an indicator of post-ingestive consequences after their initial hedonic value has acquired a positive or a negative value via previous individual food learning or evolutionary processes. Thus they represent cues that could help ruminants to anticipate the post-ingestive consequences of a food and to improve their learning efficiency, especially in complex environments. This review then suggests that food sensory characteristics could be of importance to provide pleasure to animals, increase palatability of a food, and help them learn in complex feeding situations which could improve animal welfare and productivity.

FAVREAU-PEIGNÉ A., BAUMONT R., GINANE C., Les rôles des caractéristiques sensorielles des aliments dans le comportement alimentaire des ruminants domestiques. INRA Prod. Anim., 26, 1, 25-34. 